\title{
Advising Obese Adults about Diet and Physical Activity in Sousse, Tunisia
}

\author{
Jihene Maatoug, Imed Harrabi, Sonia Hmad, Mylene Belkacem, \\ Asma Nouira, and Hassen Ghannem
}

Department of Epidemiology, University Hospital Farhat Hached, Sousse, Tunisia

Correspondence should be addressed to Jihene Maatoug; jihenmaatoug3107@gmail.com

Received 30 November 2012; Accepted 27 January 2013

Academic Editors: M. Jones and A. M. Shapiro

Copyright (C) 2013 Jihene Maatoug et al. This is an open access article distributed under the Creative Commons Attribution License, which permits unrestricted use, distribution, and reproduction in any medium, provided the original work is properly cited.

\begin{abstract}
Background. To our knowledge no study has been conducted in Tunisia to describe practice of health care providers towards chronic disease risk factors, particularly among obese adults. Aim. This study is aimed at assessing the level of giving advice on diet and physical activity by health care providers for obese adults comparing to nonobese adults in Tunisia. Methods. A crosssectional survey was carried out in 2010 for adults aged from 18 to 65 years living in the region of Sousse. The questionnaires were administered by an interview made by trained data collectors to standardize the administration of the questionnaire. Anthropometric measurements of height and weight were obtained using a standardized protocol from each participant. Results. The findings from this study indicate that obesity is frequent among adults essentially among women and aged categories. It also shows that obese adults are not adequately diagnosed with few proportion of anthropometric measure evaluation among all the participants. Even if obese participants were significantly more advised than nonobese participants with, respectively, $28.5 \%$ to lose weight and $23.8 \%$ to increase physical activity, this proportion remains low.
\end{abstract}

\section{Introduction}

Obesity has become a growing global health problem. There are approximately 937 million and 396 million obese and overweight adults worldwide, respectively [1]. Southern and eastern Mediterranean countries have been particularly affected by this evolution $[2,3]$. However, the obesity has wellknown associations with all-cause mortality $[4,5]$, morbidity [6], and disability, resulting in unhealthy life years with poor quality of life $[7,8]$ and increased health care costs $[9,10]$

The World Health Organization report on obesity states that sedentary lifestyle and consumption of high-fat energydense diets are fundamental causes of the obesity epidemic [11]. Health promotion strategies, including behavioral interventions aimed at modifying dietary habits and physical activity patterns, are essential in prevention and management of obesity. A substantial part (60-70\%) of population makes visits to their general practitioner (GP) each year [12]. However, the studies have shown low rate of counseling on lifestyle changes given to overweight patients in primary health care $[13,14]$.

Health care providers such as general practitioner, and nurses have the opportunity to confront this epidemic and to educate their patients about weight loss. Physicians and other health care professionals may, however, be missing an important opportunity to counsel overweight individuals to lose weight or to maintain their weight and prevent morbidity and mortality [15].

In recent years, there has been much research-based focus on using the general practice setting for health promotion including improving dietary and exercise practices among patients, particularly for weight management. To our knowledge no study has been conducted in Tunisia to describe practice of health care providers towards chronic disease risk factors, particularly among obese adults.

This study is aimed at assessing the level of giving advice on diet and physical activity by health care providers for obese and nonobese adults in Tunisia. 


\section{Material and Methods}

2.1. Study Design. We conducted a cross-sectional survey carried out in 2010. The target population was all adults aged from 18 to 65 years.

2.2. Study Population. The sampling frame was derived by the Tunisian National Institute of Statistics from the database of the most recent census of the population carried out in 2004. According to this list of households, we used a random representative sample of the general urban population of Sousse Jawhara, Sousse Riadh, and Msaken by selecting districts from these regions. Then, all adults aged from 18 to 65 years living in these districts and who were present at data collection were included.

2.3. Data Collection. We used an Arab pretested questionnaire to collect data about health status, chronic disease risk factors, and care received in medical consultation for participants. The questionnaires were administered by interview by trained data collectors to standardize the administration of the questionnaire.

Anthropometric measurements of height and weight were obtained using a standardized protocol from each participant during interview and clinical examination. Height was measured, to the nearest $0.5 \mathrm{~cm}$, without shoes, with the participant's back square against the wall tape, eyes looking straight ahead with a right-angle triangle resting on the scalp and against the wall. Weight was measured with a lever balance to the nearest 100 grams, without shoes, in light undergarments. Body mass index (BMI) was calculated as weight $(\mathrm{kg})$ divided by the square of the height $(\mathrm{m})$ and obesity was defined as BMI $>30 \mathrm{~kg} / \mathrm{m}_{2}$ [16].

2.4. Statistical Analysis. The mean $\pm \mathrm{SD}$ values were calculated for continuous variables and the $\%$ age of each subgroup for categorical variables. Comparisons between two group means were performed by using the $t$-test. Associations between categorical variables were tested by using contingency tables and the $\chi^{2}$ test. All data analyses were conducted using SPSS statistical software (Version 10.0). All statistical tests were 2 -tailed, and $P$ values $<0.05$ were considered statistically significant.

2.5. Ethical Consideration. The study was approved by the Ethics Committee of the University Hospital Farhat Hached, Tunisia. It does not represent any risk for participants who gave their consent before responding to the questionnaire.

\section{Results}

The response rate was $72.1 \%$. Our population was composed of 1880 adult participants with $64 \%$ of women. The mean age of participants was $37.9 \pm 13.5$ years. The prevalence of obesity was $18.1 \%$ and $33.5 \%$, respectively, among men and women.

The prevalence of obesity increased significantly with age in both sex (Table 1).
TABLE 1: Prevalence of the obesity by sex and age among adults aged from 18 to 65 years in Sousse.

\begin{tabular}{lccc}
\hline & Men $n(\%)$ & Women $n(\%)$ & $P$ \\
\hline Obesity $\left(\mathrm{BMI}>30 \mathrm{~kg} / \mathrm{m}^{2}\right)$ & $111(18.1)$ & $395(33.5)$ & $<\mathbf{0 . 0 0 1}$ \\
Age & & & \\
$\quad<30$ years & $20(7.2)$ & $48(12.0)$ & \\
$30-45$ years & $35(20.8)$ & $149(37.3)$ & $<\mathbf{0 . 0 0 1}$ \\
$>45$ years & $64(31.1)$ & $196(52.1)$ & \\
\hline
\end{tabular}

TABLE 2: Sociodemographic distribution of adults according to obesity status in the region of Sousse, Tunisia.

\begin{tabular}{lccc}
\hline & \multicolumn{3}{c}{ Obesity } \\
& No $n(\%)$ & Yes $n(\%)$ & $P$ \\
\hline $\begin{array}{l}\text { Marital status (single) } \\
\text { Level of education less }\end{array}$ & $538(40.8)$ & $50(\mathbf{9 . 7})$ & $<\mathbf{0 . 0 0 1}$ \\
than 6th grade & $365(27.6)$ & $260(50.7)$ & $<\mathbf{0 . 0 0 1}$ \\
Do not work & $189(63.0)$ & $314(61.3)$ & $<\mathbf{0 . 0 0 1}$ \\
\hline
\end{tabular}

Demographic characteristics of the study population by obesity status are provided in Table 2 . The obese participants were more likely to be married, did not work, and had a level of education less than 6th grade. In fact, only $9.7 \%$ of obese participants were single versus 40.8 single persons among nonobeses.

Only 6 obese participants (1.2\%) were diagnosed as having obesity by a health professional as reported by participants.

Among obese participants, $42.3 \%$ reported feeling good health status versus $64.3 \%$ among nonobese participants $(P<$ $\left.10^{-3}\right)$. However, there was not a significant difference between them concerning feeling stressed or under a lot of pressure, or depressed or bothered by little interest or pleasure in doing things (Table 3). Concerning chronic diseases risk factors, obese participants were more likely to have high blood pressure, hypercholesterolemia, and diabetes in their personal antecedents (Table 3).

Among obese participants, 178 (70.1\%) were screened as having high blood pressure but they were not diagnosed. This proportion was $82.9 \%$ (281 participants) among nonobese participants.

The proportion of obese adults who had visited a physician in the previous twelve months was $74.3 \%$ versus $64.8 \%$ among nonobese participants with significant difference $(P<$ $\left.10^{-3}\right)$.

Among obese adults who had visited a physician in the previous twelve months, an estimated $30.9 \%$ reported that their weight was taken by a health care provider versus $25.5 \%$ among nonobese adults $(P=0.05)$ (Table 4$)$. Hip and waist circumference were taken at almost the same proportion for obese and nonobese consultants with, respectively, 5.5\% and $7.1 \%$ among obese and $5.2 \%$ and $4.8 \%$ among nonobese (Table 4). Blood pressure was taken in $67.4 \%$ and $50.1 \%$ of cases respectively for obese and nonobese adults $\left(P<10^{-3}\right)$. Respectively, blood cholesterol and blood glucose were taken significantly more frequently among obese consultants. 
TABLE 3: Health status and antecedents of adults according to obesity status in the region of Sousse, Tunisia.

\begin{tabular}{|c|c|c|c|}
\hline & \multicolumn{3}{|c|}{ Obesity } \\
\hline & No $n(\%)$ & Yes $n(\%)$ & $P$ \\
\hline Feel good health status & $694(64.3)$ & $187(42.3)$ & $<0.001$ \\
\hline Feel stressed or under a lot of pressure & $286(21.6)$ & $131(25.5)$ & 0.07 \\
\hline Feel depressed & $549(41.6)$ & $209(40.7)$ & 0.71 \\
\hline bothered by little interest or pleasure in doing things & $528(40.0)$ & $199(38.7)$ & 0.62 \\
\hline Have diagnosis of high blood pressure & $69(5.2)$ & $97(18.9)$ & $<0.001$ \\
\hline Have diagnosis of high cholesterol & $26(2.0)$ & $44(8.6)$ & $<0.001$ \\
\hline Have diagnoses of diabetes & $50(3.8)$ & $62(12.1)$ & $<0.001$ \\
\hline
\end{tabular}

TABLE 4: Distribution of tests and/or measurements taken by a health care provider at any time in the past 12 months among adults by obesity status in the region of Sousse, Tunisia.

\begin{tabular}{|c|c|c|c|}
\hline & \multicolumn{3}{|c|}{ Obesity } \\
\hline & No $n(\%)$ & Yes $n(\%)$ & $P$ \\
\hline $\begin{array}{l}\text { Have you visited a health care facility in the past } 12 \text { months for your routine health } \\
\text { care }\end{array}$ & $856(64.8)$ & $382(74.3)$ & $<0.001$ \\
\hline Weight was taken by a health care provider at any time in the past 12 months & $217(25.5)$ & $117(30.9)$ & 0.05 \\
\hline Height was taken by a health care provider at any time in the past 12 months & $138(16.2)$ & $74(19.5)$ & 0.15 \\
\hline $\begin{array}{l}\text { Hip circumference was taken by a health care provider at any time in the past } \\
12 \text { months }\end{array}$ & $44(5.2)$ & $21(5.5)$ & 0.79 \\
\hline $\begin{array}{l}\text { Waist circumference was taken by a health care provider at any time in the past } \\
12 \text { months }\end{array}$ & $41(4.8)$ & $27(7.1)$ & 0.11 \\
\hline $\begin{array}{l}\text { Blood cholesterol was taken by a health care provider at any time in the past } \\
12 \text { months }\end{array}$ & $203(23.9)$ & $169(44.7)$ & $<0.001$ \\
\hline Blood pressure was taken by a health care provider at any time in the past 12 months & $427(50.1)$ & $256(67.4)$ & $<0.001$ \\
\hline Blood glucose was taken by a health care provider at any time in the past 12 months & $240(28.2)$ & $193(51.1)$ & $<0.001$ \\
\hline
\end{tabular}

The proportion of obese adults who had visited a physician in the previous twelve months reported that a health care provider discussed with them their weight status in $30.4 \%$ of cases and being advised to lose weight in $28.5 \%$ of cases (Table 5). They received advice about how to follow a healthy diet in $28.6 \%$ versus $15.7 \%$ for nonobese participants with significant difference. Health care providers gave advice or treatment to increase physical activity in $23.8 \%$ and $11.7 \%$, respectively, for obese and nonobese participants. However, they discussed with their patients their risk for diabetes, cardiovascular disease (CVD), and/or cancer only in almost $4 \%$ of cases for the two groups (Table 5 ).

\section{Discussion}

The findings from this study indicate that obesity is frequent among adults essentially among women and aged categories. It also shows that obese adults are not adequately diagnosed with few proportion of anthropometric measure evaluation among all the participants.

Even if obese participants were significantly more advised than nonobese participants, this proportion remains low. Other studies that evaluated counseling obese patients by GPs have shown that proportion of advised persons varies from $5 \%$ to more than $40 \%[13,14]$. In the USA [17] $39 \%$ of obese adults who had visited their GP in the previous 12 months were advised to lose weight. In another study, 34\% reported being counseled about exercise at their last visit and patients who were overweight to obese were more likely to be counseled [18].

Sciamanna et al. [15] revealed that advice to lose weight is uncommon and is given primarily to those who are already obese. Only about $17 \%$ in this study, individuals who have visited a physician and who have health care coverage indicated receiving physician's advice to lose weight even though $38 \%$ of these individuals are overweight and $24 \%$ are obese.

The rates of obesity and overweight in our study were similar to a nation-wide representative sample of Tunisian [19]. GPs may not offer lifestyle advice as they view it as ineffective, although GPs appear to agree that nutrition is important in managing disease [20]. GPs often face many barriers to offer lifestyle advice to patients, particularly if the presenting problem is unrelated. GPs may lack confidence in offering more detailed nutrition advice [21] due, in part, to insufficient knowledge [22]. Several studies have shown that GPs knowledge about nutrition and physical activity in management of obesity is incomplete [23]. They expressed the need for clinical guidelines and supplementary training. Even when GPs have nutritional knowledge, they find it difficult to communicate this knowledge effectively. GPs would therefore benefit from additional training in counseling skills. 
TABLE 5: Distribution of advices, and treatments given by a health care provider at any time in the past 12 months among adults by obesity status in Sousse, Tunisia.

\begin{tabular}{|c|c|c|c|}
\hline & \multicolumn{3}{|c|}{ Obesity } \\
\hline & No $n(\%)$ & Yes $n(\%)$ & $P$ \\
\hline Discuss with you your current weight status? & $94(11.0)$ & $116(30.4)$ & $<0.001$ \\
\hline Give you advice or treatment to lose weight? & $72(8.4)$ & $109(28.5)$ & $<0.001$ \\
\hline Discuss with you your current diet? & $139(16.3)$ & $106(27.7)$ & $<0.001$ \\
\hline Give you advice about how to follow a healthy diet? & $134(15.7)$ & $109(28.6)$ & $<0.001$ \\
\hline Discuss with you your current level of physical activity? & $107(12.5)$ & $95(24.9)$ & $<0.001$ \\
\hline Give you advice or treatment to increase physical activity? & $100(11.7)$ & $91(23.8)$ & $<0.001$ \\
\hline $\begin{array}{l}\text { Discuss with you your risk for diabetes, cardiovascular disease } \\
\text { (CVD), and/or cancer? }\end{array}$ & $39(4.6)$ & $18(4.7)$ & 0.92 \\
\hline
\end{tabular}

Other barriers include time constraints, a lack of incentives or reimbursements [24], complexity of advice, lack of training in counseling skills, a lack of interest [25], the idea that patients are not motivated [21], and a long delay between intervention and observable effects [26].

Diet and physical activity are relatively time consuming to assess; there is no simple measurement tool, and results are open to misinterpretation by patients. The authors found that more GPs reported usually "offering advice" than "assessing readiness to change," which suggests that patients may have been receiving advice that they were not ready to listen to or act upon. Routine assessment of readiness to change before offering suggestions or advice may yield better results for lifestyle counseling [27].

Therefore, a discrepancy seems to exist between the recommendations patients report to have received from their healthcare professionals and clinical guidelines, as well as findings from research studies on the prevalence and content of weight loss advice [28].

Whatever the exact reasons for the low propensity to give advice, it is imperative that physicians and other health care professionals are encouraged through various programs or approaches (training about obesity, intervention strategies, public policy, etc.) to do more to advice their overweight and obese patients about weight loss [29].

\section{Conclusion}

Chronic disease risk factors are common in the Tunisian population, and health care providers are ideally placed to offer assessment, advice, and referral to services and programs. Future studies that would determine the predictive factors of counseling patients to lose weight and directly measure the effect of physician's advice on actual weight or behavior over time are needed to address this issue further.

\section{Acknowledgment}

This project is funded by UnitedHealth Group through The NHLBI and UnitedHealth Chronic Disease Initiative.

\section{References}

[1] T. Kelly, W. Yang, C. S. Chen, K. Reynolds, and J. He, "Global burden of obesity in 2005 and projections to 2030," International Journal of Obesity, vol. 32, pp. 1431-1437, 2008.

[2] A. O. Musaiger, "Overweight and obesity in eastern mediterranean region: prevalence and possible causes," Journal of Obesity, vol. 2011, Article ID 407237, 17 pages, 2011.

[3] C. Papandreou, T. AbuMourad, C. Jildeh, Z. Abdeen, A. Philalithis, and N. Tzanakis, "Obesity in Mediterranean region (1997-2007): a systematic review," Obesity Reviews, vol. 9, pp. 389-399, 2008.

[4] A. J. Cameron, D. J. Magliano, J. E. Shaw et al., "The influence of hip circumference on the relationship between abdominal obesity and mortality," International Journal of Epidemiology, vol. 41, pp. 484-494, 2012.

[5] G. Whitlock, S. Lewington, P. Sherliker et al., "Body-mass index and cause-specific mortality in 900000 adults: collaborative analyses of 57 prospective studies," The Lancet, vol. 373, no. 9669, pp. 1083-1096, 2009.

[6] R. F. Grimble, "The true cost of in-patient obesity: impact of obesity on inflammatory stress and morbidity," Proceedings of the Nutrition Society, vol. 69, no. 4, pp. 511-517, 2010.

[7] T. G. K. Bentley, M. Palta, A. J. Paulsen et al., "Race and gender associations between obesity and nine health-related quality-oflife measures," Quality of Life Research, vol. 20, no. 5, pp. 665674, 2011

[8] M. Perez and C. S. Warren, "The relationship between quality of life, bingeeating disorder, and obesity status in an ethnically diverse sample," Obesity, vol. 20, pp. 879-885, 2012.

[9] J. P. Moriarty, M. E. Branda, K. D. Olsen et al., "The effects of incremental costs of smoking and obesity on health care costs among adults: a 7-year longitudinal study," Journal of Occupational and Environmental Medicine, vol. 54, pp. 286-291, 2012.

[10] J. Cawley and C. Meyerhoefer, "The medical care costs of obesity: an instrumental variables approach," Journal of Health Economics, vol. 31, pp. 219-230, 2012.

[11] Report of WHO Consultation on Obesity: Obesity: Preventing and Managing the Global Epidemic, World Health Organization, Geneva, Switzerland, 1998. 
[12] R. Grol, M. Wensing, J. Mainz et al., "Patients' priorities with respect to general practice care: an international comparison," Family Practice, vol. 16, no. 1, pp. 4-11, 1999.

[13] J. Huang, H. Yu, E. Marin, S. Brock, D. Carden, and T. Davis, "Physicians' weight loss counselling in two public hospital primary care clinics," Academic Medicine, vol. 79, no. 2, pp. 156161, 2004.

[14] M. J. Duaso and P. Cheung, "Health promotion and lifestyle advice in a general practice: what do patients think?" Journal of Advanced Nursing, vol. 39, no. 5, pp. 472-479, 2002.

[15] C. N. Sciamanna, D. F. Tate, W. Lang, and R. R. Wing, "Who reports receiving advice to lose weight? Results from a multistate survey," Archives of Internal Medicine, vol. 160, no. 15, pp. 2334-2339, 2000.

[16] National Institutes of Health, "Clinical Guidelines on the Identification, Evaluation, and Treatment of Overweight and Obesity in Adults-The Evidence Report," NIH, 1998.

[17] J. Y. Ko, D. R. Brown, D. A. Galuska, J. Zhang, H. M. Blanck, and B. E. Ainsworth, "Weight loss advice U.S. obese adults receive from health care professionals," Preventive Medicine, vol. 47, no. 6, pp. 587-592, 2008.

[18] C. C. Wee, E. P. McCarthy, R. B. Davis, and R. S. Phillips, "Physician counseling about exercise," Journal of the American Medical Association, vol. 282, no. 16, pp. 1583-1588, 1999.

[19] J. El Ati, P. Traissac, F. Delpeuch et al., "Gender obesity inequities are huge but differ greatly according to environment and socio-economics in a north african setting: a national crosssectional study in Tunisia," PLoS One, vol. 7, no. 10, Article ID e48153, 2012.

[20] S. E. Morris, M. E. J. Lean, C. R. Hankey, and C. Hunter, "Who gets what treatment for obesity? A survey of GPs in Scotland," European Journal of Clinical Nutrition, vol. 53, supplement 2, pp. S44-S48, 1999.

[21] J. L. Buttriss, "Food and nutrition: attitudes, beliefs, and knowledge in the United Kingdom," American Journal of Clinical Nutrition, vol. 65, no. 6, pp. 1985-1995, 1997.

[22] L. G. Nicholas, C. D. Pond, and D. C. Roberts, "Dietitiangeneral practitioner interface: a pilot study on what influences the provision of effective nutrition management," The American Journal of Clinical Nutrition, vol. 77, no. 4, pp. 1039-1042, 2003.

[23] S. A. Flocke, A. Clark, K. Schlessman, and G. Pomiecko, "Exercise, diet, and weight loss advice in the family medicine outpatient setting," Family Medicine, vol. 37, no. 6, pp. 415-421, 2005.

[24] G. D. Foster, T. A. Wadden, A. P. Makris et al., "Primary care physicians' attitudes about obesity and its treatment," Obesity Research, vol. 11, no. 10, pp. 1168-1177, 2003.

[25] A. Coulter and T. Schofield, "Prevention in general practice: the view of doctors in the Oxford region," British Journal of General Practice, vol. 41, no. 345, pp. 140-143, 1991.

[26] J. J. van Binsbergen and A. J. Drenthen, "Patient information letters on nutrition: development and implementation," The American Journal of Clinical Nutrition, vol. 77, no. 4, pp. 10351038, 2003.

[27] E. Denney-Wilson, M. Fanaian, Q. Wan, S. Vagholkar, H. Schütze, and M. Harris, "Lifestyle risk factors in general practice: routine assessment and management," Australian Family Physician, vol. 39, no. 12, pp. 950-953, 2010.

[28] J. Y. Ko, D. R. Brown, D. A. Galuska, J. Zhang, H. M. Blanck, and B. E. Ainsworth, "Weight loss advice U.S. obese adults receive from health care professionals," Preventive Medicine, vol. 47, no. 6, pp. 587-592, 2008.
[29] M. L. Loureiro and R. M. Nayga Jr., "Obesity, weight loss, and physician's advice," Social Science \& Medicine, vol. 62, no. 10, pp. 2458-2468, 2006. 


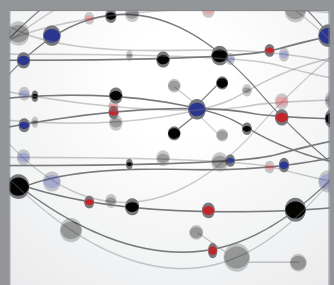

The Scientific World Journal
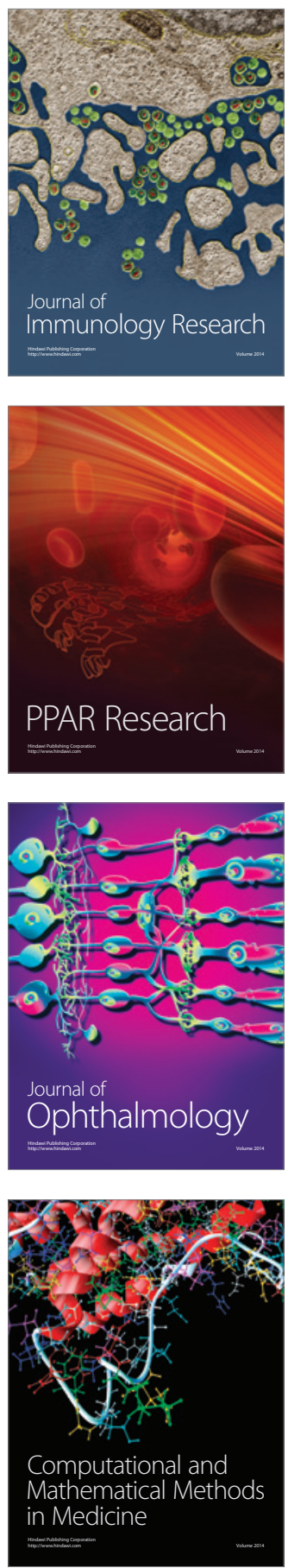

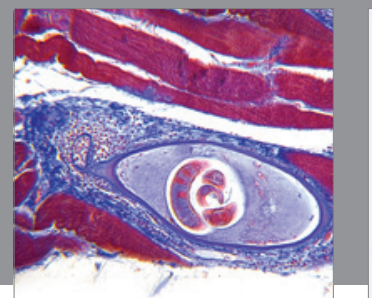

Gastroenterology

Research and Practice
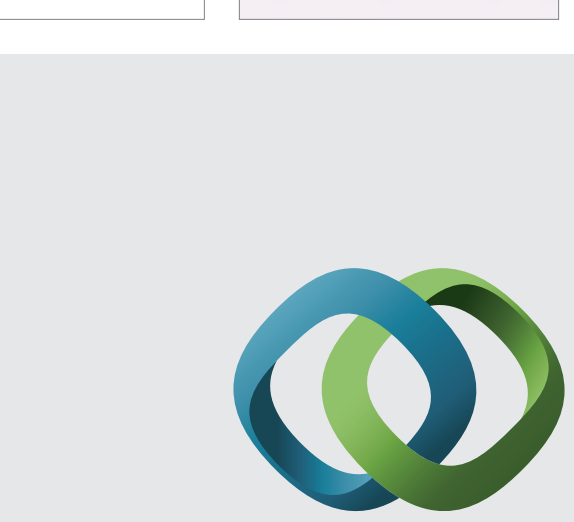

\section{Hindawi}

Submit your manuscripts at

http://www.hindawi.com
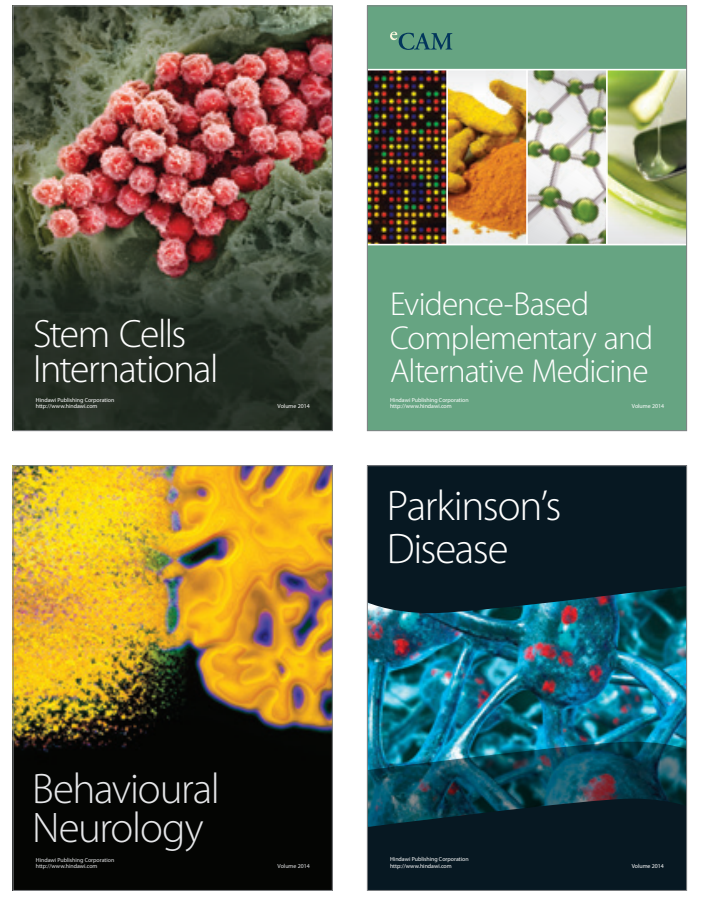
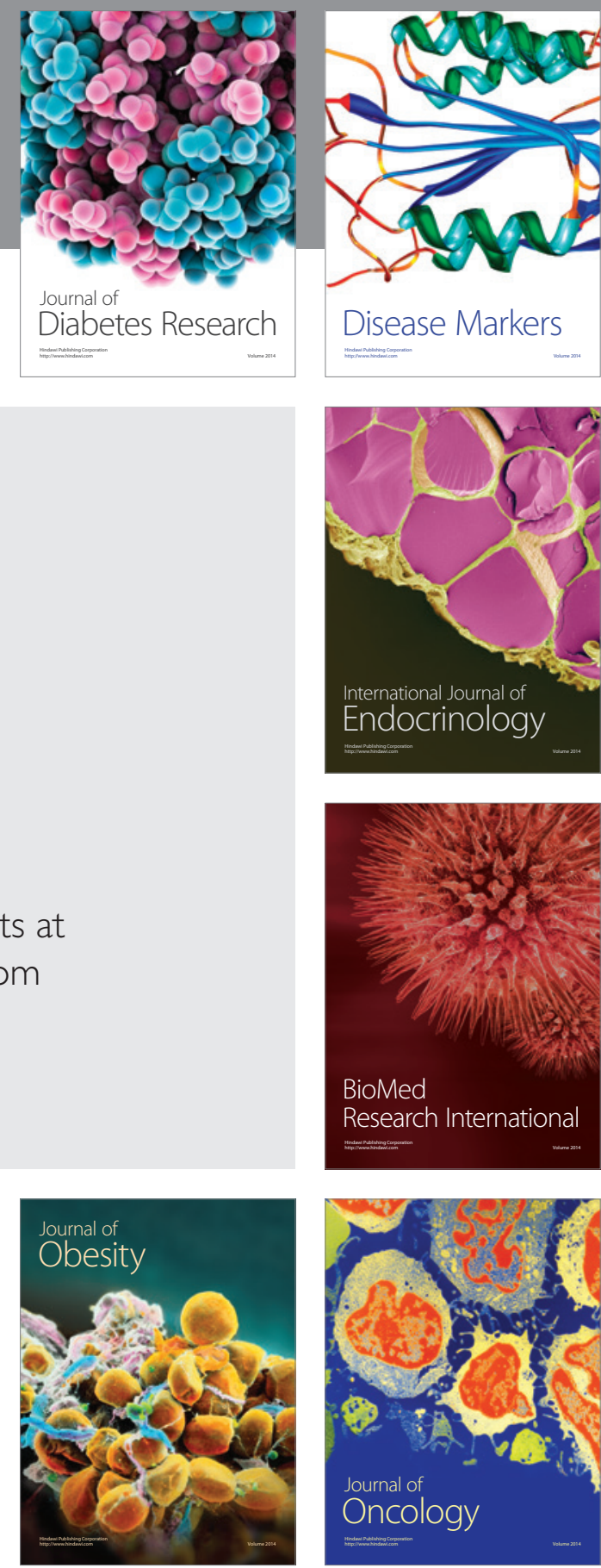

Disease Markers
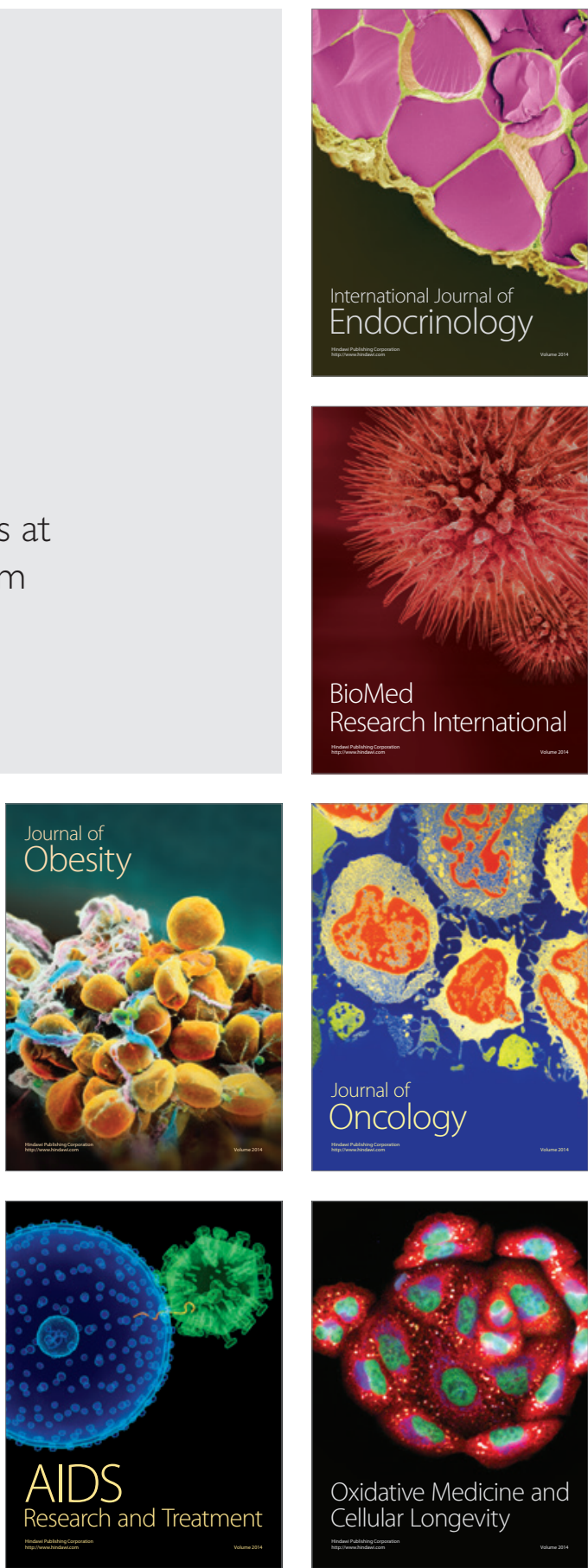